\title{
The Ejecta of Classical Novae
}

\author{
T.J. O'Brien, R.J. Davis
}

Jodrell Bank Observatory, The University of Manchester, Macclesfield, SK11 9DL, UK

\author{
M.F. Bode, S.P.S. Eyres, J.M. Porter \\ Astrophysics Research Institute, Liverpool JMU, Birkenhead, CH41 \\ $1 L D, U K$
}

\begin{abstract}
Classical novae are interacting binary stars in which a thermonuclear runaway in material accreted onto a white dwarf from a companion red dwarf results in the ejection of around $10^{-4} M_{\odot}$ at hundreds to thousands of kilometres per second. Recent Hubble Space Telescope and MERLIN imaging of the expanding ejecta from several classical novae are presented. In general the ejecta are clumpy but often display coherent structures, most notably equatorial rings of enhanced emission encircling prolate ellipsoidal shells. Physical mechanisms (including the common envelope phase and anisotropic irradiation of the shell) which may result in the generation of these structures are discussed.
\end{abstract}

\section{Introduction}

The central systems of classical novae consist of a white dwarf-red dwarf pair in a close binary orbit (orbital periods are typically a few hours). Hydrogenrich material is transferred from the cool star onto the compact object via an accretion disc. Over thousands of years the pressure at the base of this accreted envelope builds up until thermonuclear reactions begin under degenerate conditions. The ensuing thermonuclear runaway and accompanying increase in bolometric luminosity results in the ejection of around $10^{-5}$ to $10^{-4} M_{\odot}$ of material at velocities of the order of hundreds to thousands of kilometres per second. The ejecta are composed of some fraction (not necessarily all) of the accreted envelope enhanced by the products of the thermonuclear runaway and possibly mixed with some white dwarf matter. The expanding shell can typically be resolved a few months after outburst using high resolution imaging at radio or optical wavelengths. Old nova shells remain visible years after outburst as they are bathed in the photoionizing radiation field of the hot central white dwarf with its re-established accretion disc. Such outbursts are thought to repeat every 10,000 to 100,000 years and may be repeated several hundred times for a given system. 


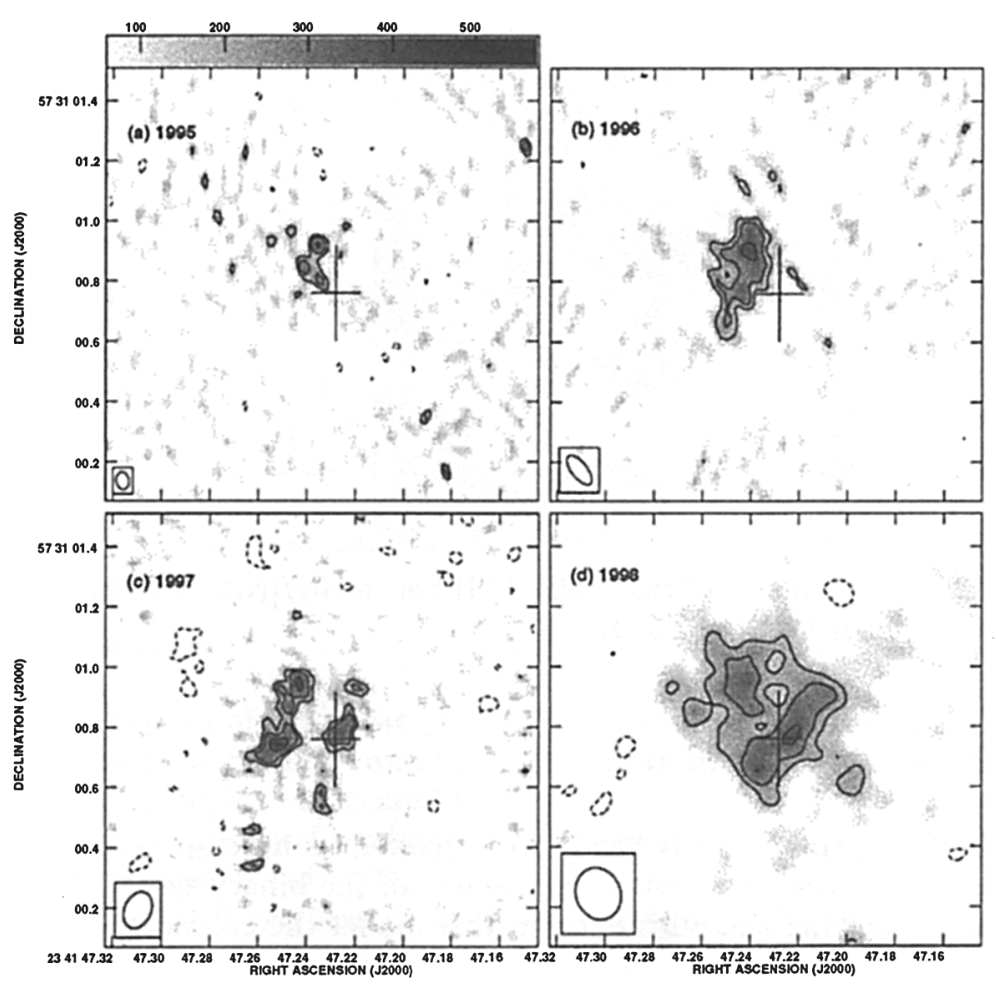

Figure 1. 6cm-radio images of the expanding shell of V705 Cas made with the MERLIN array (Eyres et al 2000).

\section{Optical \& radio imaging}

The ejected shells are often neither smooth nor spherically symmetric. Our radio imaging of several recent novae using the MERLIN array shows evolving clumpy structures which indicate that optical depth effects and departures from spherical symmetry play a major role. Figure 1 shows the evolution of the shell of V705 Cas (Nova Cas 1993, Eyres et al 2000). Similar structures which develop asymmetrically are seen in our monitoring of V723 Cas (Nova Cas 1995, O'Brien et al, in prep.).

Our ground-based optical imaging survey (Gill \& O'Brien 1998; Slavin, O'Brien, \& Dunlop 1995) which was followed up by Hubble Space Telescope imaging and ground-based spectroscopy (Gill \& O'Brien 2000) revealed a number of examples of coherent structures in old nova shells. The most notable of these were equatorial rings of enhanced emission circling the waist of prolate ellipsoidal shells, see Figure 2 for the example of nova FH Ser. Similar rings are seen in, for example, the shells of V533 Her, DQ Her and RR Pic. Long-slit spectra of FH Ser (Gill \& O'Brien 2000) and DQ Her (O'Brien \& Gill, in preparation) revealed that these rings seen in $\mathrm{H} \alpha /[\mathrm{NII}]$ images were due to emission in [NII] rather than $\mathrm{H} \alpha$. The presence of similar features is suggested in nova V705 Cas (Figure 1) on the basis of spatially unresolved spectroscopy taken nearer to outburst. 


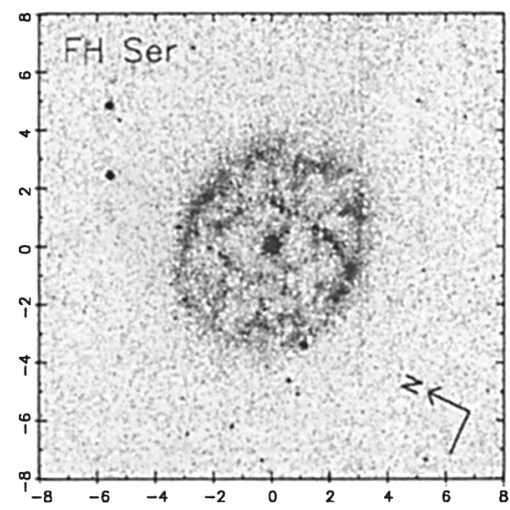

Figure 2. HST image of the shell of $\mathrm{FH}$ Ser in $\mathrm{H} \alpha /[\mathrm{NII}]$, axes marked in $\operatorname{arcsec}$ (Gill \& O'Brien 2000).

\section{The [NII]-ring phenomenon}

The generation of prolate shells has been suggested as a possible result of the common-envelope phase in which the ejecta engulf the binary system for an extended period following the outburst. In this model the secondary star heats and spins up the ejecta driving mass-loss into the equatorial plane (e.g. Shankar, Livio, \& Truran 1991). It was thought that this might naturally result in equatorial rings formed by density enhancements but further simulations have shown that this is not so straightforward (Porter, O'Brien, \& Bode 1998). In any case, our recent observations of these systems show that a simple density enhancement can not in fact explain the observed rings.

There are a number of mechanisms which may result in the production of [NII]-enhanced rings. For example, if [NII] emission is suppressed by collisional de-excitation in the major part of the shell then a density decrease in the equatorial region could produce a relative increase in emission in the form of a ring. Another possibility is that the relative abundance of nitrogen is increased in the equatorial region resulting in enhanced [NII] emission. This could be due to dredge-up of material from the white dwarf prior to outburst via a mechanism which is more efficient at low white dwarf latitudes. A third possibility is an effect due to variations in the photoionizing radiation field. Old nova shells are illuminated by the central system comprising a hot white dwarf and accretion disc. Either because the accretion disc will present a different aspect when viewed from different angles or because emission from the white dwarf is obscured by the disc, the irradiation is likely to be largest at the poles of the shell and least at the equator. One possibility for explaining increased [NII] emission around the shell's equator is that nitrogen is doubly ionized or above in those regions of the shell at higher latitudes which see more intense or energetic radiation and hence [NII] emission is suppressed. We have investigated this option using simple prescriptions for this aspherical illumination which demonstrate that this may be a viable explanation. Figure 3 shows the results of one such calculation using the photoionization code Cloudy (Ferland 1996). 


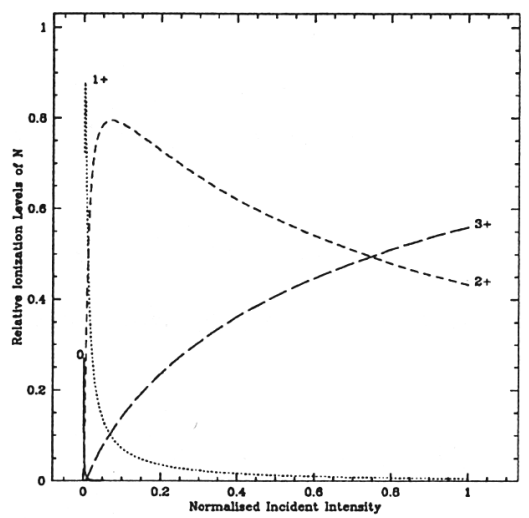

Figure 3. Nitrogen ionization states as a function of illumination.

\section{Conclusions}

The nebular ejecta of old novae provide important information on the nature of the thermonuclear runaway and the parameters of the binary progenitor. The ejecta are often highly structured with equatorial rings which, in several novae, are enhanced in emission from [NII]. We are now obtaining high-resolution images of these structures in both the optical and radio regimes but progress needs to be made in reconciling these data. Nebular shaping mechanisms including the effects of the binary companion and the rotation and magnetic field of the white dwarf require further exploration. A new suggestion is that photoionization models of the shells should allow for the variation in illumination around the shell resulting from shadowing by the accretion disc. These models should be constrained by spectrophotometry in the optical/uv parts of the spectrum.

\section{References}

Eyres, S. P. S., Bode, M. F., O'Brien, T. J., Watson, S. K., Davis, R. J. 2000, MNRAS, 318, 1086

Ferland, G. J. 1996, http://nimbus.pa.uky.edu/cloudy/, Internal Report, University of Kentucky

Gill, C. D., O'Brien, T. J. 1998, MNRAS, 300, 221

Gill, C. D., O'Brien, T. J. 2000, MNRAS, 314, 175

Porter, J. M., O'Brien, T. J., Bode, M. F. 1998, MNRAS, 296, 943

Slavin, A. J., O'Brien, T. J., Dunlop, J. S. 1995, MNRAS, 276, 353

Shankar, A., Livio, M., Truran, J. W. 1991, ApJ, 374, 693 